Ciencia y Educación, Vol. 6, No. 1, enero-abril, 2022

ISSN (impreso): 2613-8794 • ISSN (en línea): 2613-8808

DOI: https://doi.org/10.22206/cyed.2022.v6i1.pp27-42

\title{
Aprendizajes de los profesores sobre la docencia en una sociedad conectada
}

\author{
Teachers' learning about teaching in a connected society \\ Joana Romanowski ${ }^{a}$ ORCID: 0000-0001-7043-5534 \\ Simone Cartaxo ${ }^{\mathrm{b}}$ ORCID: 0000-0002-8670-6324 \\ Liliamar Hoça ${ }^{c}$ ORCID: 0000-0002-9650-0215 \\ Pura Martins ${ }^{\mathrm{d}}$ ORCID: 0000-0003-0300-8318 \\ Ademir Mendes ${ }^{e}$ ORCID: 0000-0003-4929-9544
}

Recibido: 18/07/2021・Aprobado: 6/09/2021

Cómo citar: Romanowski, J. P., Manosso Cartaxo, S. R., Hoça, L., Martins, P., \& Mendes, A. Aprendizajes de los profesores sobre la docencia en una sociedad conectada. Ciencia y Educación, 6(1), 27-42. https://doi.org/10.22206/cyed.2022.v6i1.pp27-42

\section{Resumen}

El artículo tiene como problema: ¿cómo aprenden los docentes en una sociedad conectada? El objetivo es conocer cómo son desarrollados los procesos de aprendizaje de los docentes, en una sociedad conectada que les ofrece alternativas, formales e informales, para mejorar su conocimiento profesional. La investigación tiene un enfoque cualitativo y los datos fueron obtenidos a través de un cuestionario respondido por 245 docentes de todas las regiones de Brasil. Los hallazgos indican que los docentes aprenden, en la práctica, mediante investigación, de manera interactiva en grupos colaborativos y a través de procesos de autoformación. Se evidencia que la formación docente se produce a través del aprendizaje formal e informal, y que estos aprendizajes se asocian a su formación y desarrollo profesional, y elevan el estatus de profesionalización de los docentes.

Palabras clave: aprendizaje de profesores; práctica pedagógica; formación de profesores; desarrollo profesional docente; aprendizaje informal.

\begin{abstract}
The article has as a problem: how do teachers learn in a connected society? The objective is to know how the learning processes of teachers are developed, in a connected society that offers them alternatives, formal and informal, to improve their professional knowledge. The qualitative approach research and data were obtained through a questionnaire and answered by 245 teachers from all regions of Brazil. The findings indicate that teachers learn in practice, through research, interactively in collaborative groups, and through self-training processes. It is evident that teacher education occurs through formal and informal learning, and that these learnings are associated with their preparation and professional development and raise the professional status of teachers.
\end{abstract}

Keywords: Teacher learning; pedagogical practice; teacher education; teacher professional development; informal learning.

\footnotetext{
a Centro de la Universidad Internacional (UNINTER), Brasil. Correo-e: joana.r@uninter.com

b Universidad Estatal de Ponta Grossa (UEPG), Brasil. Correo-e: srmcartaxo@uepg.br

c Rede Municipal de Ensino de Curitiba, Brasil. Correo-e: liliamar.hoca@unicesumar.edu.br

d Pontifícia Universidade Católica do Paraná, Brasil. Correo-e: pura.oliver@pucpr.br

e Centro de la Universidad Internacional (UNINTER), Brasil. Correo-e: ademir.m@uninter.com
} 


\section{Introducción}

La formación docente es un proceso que tiene lugar desde la escolarización, en los cursos de formación, y a lo largo de la carrera que involucra las distintas etapas que Vaillant \& Marcelo (2016) sistematizan como a, b, c y d: antecedentes, formación básica, formación continua y desarrollo profesional. A partir de esa perspectiva, este artículo problematiza los aprendizajes docentes de los profesores de educación básica. Esta investigación forma parte del proyecto titulado ¿Cómo aprenden los profesores en una sociedad conectada?, aprobado por el Plan Estatal 2017-2020, código PGC2018-096474-B-I00", coordinada por Carlos Marcelo García, de la Universidad de Sevilla, con financiación del Ministerio de Economía, Industria y Competitividad de España.

El aprendizaje docente constituye uno de los ejes fundamentales del proceso de formación de profesores, sin embargo, ha sido poco investigado. El objetivo de la investigación es conocer cómo se desarrollan los procesos de aprendizaje de los docentes del siglo XXI, en una sociedad conectada que ofrece formas alternativas, formales e informales, de mejorar el conocimiento profesional de los docentes. También se propone analizar y comprender el papel de las tecnologías digitales como contextos que crean condiciones para facilitar el desarrollo del liderazgo informal, que permite a los docentes adquirir, compartir, aplicar y transferir la práctica profesional de los saberes docentes. Esta investigación se propone contribuir a la comprensión de las dimensiones expresadas en el aprendizaje de los docentes, en la formación formal e informal.

El supuesto es que "las interacciones entre las personas, en contextos de aprendizaje, contribuyen al desarrollo complejo de los individuos, que forman y se forman" (Vaillant \& Marcelo, 2016, p. 17), es decir, los profesores, al ejercer la práctica docente, enseñan y aprenden, parafraseando a Freire (1996).

En la actualidad, los tiempos y los espacios de aprendizaje se amplían, ya que estamos inmersos en una sociedad digital, caracterizada por la conectividad, la velocidad y la omnipresencia, tanto para el acceso a la información como en las interacciones laborales y sociales, provocando profundos cambios en la vida de las personas y sus prácticas, como sostiene Castells (2020).

En lasúltimas décadas sehan formulado una cantidad notable de políticas orientadas hacia la formación de docentes, además de establecerse un intenso debate sobre los diversos enfoques para preparar y apoyar a los docentes. En el contexto internacional, DarlingHammond et al. (2009) destacan que esta intensificación se manifiesta a mediados de la década de 1980 con el informe Carnegie Task Force on Teaching as a Profession, o Holmes Group, en 1986, y la fundación de la National Board for Professional Teaching Standards (NBPTS), en 1987. En Brasil, en el mismo período, se establecen discusiones en pro de la formación docente y con la ley No 9394/96 de Directrices y Bases de la Educación Nacional se define un nuevo nivel de formación mínima para el ejercicio de la enseñanza, con un cambio en las políticas de formación. Tras la aprobación de esta ley, los marcos normativos han sido intensamente discutidos en diversos órganos, especialmente en el Consejo Nacional de Educación-CNE, en asociaciones de investigación como la Asociación Nacional de Posgraduación e Investigación (ANPEd), la Asociación Nacional para la Formación de Profesionales delaEducación (ANFOPE), la Asociación Nacional de Política y Administración de la Educación (ANPAE), y la Fundación Carlos Chagas (FCC). Muchas investigaciones se realizaron para tesis y disertaciones en los programas de posgrado en educación, como se ve en los estudios de André (2002), Brzezinski (2006, 2014), Romanowski (2018) y Cartaxo \& Romanowski (2019). En estos estudios hay poca investigación sobre el aprendizaje de los docentes y la formación formal e informal, por lo que la formación en los cursos institucionalizados es reconocida como la más importante por las políticas públicas.

Destacamos la intensa oferta de cursos de profesorado en las instituciones de educación superior, públicas y privadas, en las modalidades de cursos presenciales y a distancia. Como resultado de las exigencias legales y de la intensa oferta de cursos, hay un cambio significativo en el estado de la formación del profesorado. Antes de la aprobación de la ley de Directrices y Bases de la Educación Nacional -Ley 9394/96- la formación de profesores de educación 
básica se ofrecía, en su mayoría, en el nivel medio y los últimos datos, correspondientes al año 2020, muestran que el $87 \%$ tiene una formación de nivel superior, lo que expresa un aumento en el nivel de educación de los profesores de educación básica. La concentración de la formación en el nivel superior ofrece la posibilidad de una formación sólida, favorece un mejor rendimiento de los profesores y amplía sus posibilidades de reflexión y aprendizaje, como indican Vaillant \& Marcelo (2012). Pero los docentes también amplían y profundizan su formación durante el transcurso de su desarrollo profesional, aprenden más allá de la formación académica, científica, técnica y práctica de la profesión, y también lo hacen de manera formal e informal.

Según Marcelo (2009), el aprendizaje informal contribuye a la composición de la identidad docente. Palhares (2009), al proponer una discusión sobre la educación formal y no formal, cuestiona la intensificación de la escolarización como exclusiva para la educación social, es decir, el autor reconoce que la educación escolar está atravesada por otros aprendizajes, no formales, que pueden potenciar el aprendizaje formal. Gohn (2006) discute sobre la Educación formal e informal y propone para ellas las siguientes categorías: Educación para la Ciudadanía; Justicia social; Derechos (humanos, sociales, políticos, culturales, etc.); Libertad; Igualdad y Democracia, que incluye la formación social, cultural, política y ética. Guimarães \& Faria-Fortecoëf (2021) infieren que el aprendizaje, a lo largo de la vida, favorece el saber aprender, el saber comprender y el saber proyectarse.

El estudio que presentamos comenzó con la administración de un cuestionario a profesores de enseñanza básica de todas las regiones brasileñas ${ }^{1}$. Este instrumento permitió inferir los aprendizajes de los profesores, adquiridos de manera formal e informal, y, luego de analizadas las respuestas, se establecieron las dimensiones a través de las cuales estos se realizan: en la práctica docente, en la investigación, en prácticas de enseñanza colectiva, por la autoformación. Estos aprendizajes expresan el compromiso con su formación y el interés por ampliar sus conocimientos, mejorar sus prácticas, colaborar con el aprendizaje de sus alumnos y ayudar a sus compañeros de trabajo.
El artículo está compuesto por una introducción, seguida por la descripción de la metodología del estudio, donde se detalla el proceso de recolección de datos y la caracterización de los participantes. Posteriormente, se presentan las categorías que indican las dimensiones de los aprendizajes de los profesores de educación básica surgidas del análisis y comparación de los datos. Al finalizar, hacemos una serie de consideraciones, de carácter provisorio, que expresan las inferencias derivadas de la investigación.

\section{Metodología de la investigación}

El objetivo de la investigación es conocer cómo son desarrollados los procesos de aprendizaje de los docentes, en una sociedad conectada que les ofrece alternativas, formales e informales, para mejorar su conocimiento profesional. La investigación es de enfoque cualitativo y los datos fueron obtenidos a través de un cuestionario.

La metodología de investigación de abordaje cualitativo considera fundamentales las manifestaciones de los sujetos, ya que los estudios sobre lo humano-social y lo humano-educativo requieren "una inmersión en interacciones situacionales”, como lo explican Gatti \& André (2010, p. 29). Así, es posible interpretar y comprender acercándose a lo real, de manera coherente con las formas humanas de actuar, pensar, expresar, etc.

La muestra de profesores participantes en la investigación es de 245. La recepción de los cuestionarios en línea permitió una estratificación de la muestra, tal como se describe a continuación.

\section{Caracterización de los docentes participantes en el estudio}

Con relación a la distribución geográfica de los encuestados, la mayoría, 173 (70,8\%), son profesores de la región Sur de Brasil, y en menor número de las otras regiones, Centro Oeste, 18 (7,4\%); Noreste, 15 $(6,2 \%)$; Sudeste, $35(14,4 \%)$ y Norte, con la menor cantidad de participantes, 4 . Estos datos se sistematizan en la Tabla 1. De hecho, aunque la distribución no es equivalente en las diferentes regiones, es posible 
afirmar que se obtuvieron datos de todas las regiones de Brasil.

\section{Tabla 1}

Distribución del número de participantes por regiones brasileñas

\begin{tabular}{lcc}
\hline Región & Número & $\%$ \\
\hline Centro-Oeste & 18 & $7,4 \%$ \\
\hline Noreste & 15 & $6,2 \%$ \\
\hline Sudeste & 35 & $14,4 \%$ \\
\hline Norte & 4 & $0,2 \%$ \\
\hline Sur & 173 & $70,8 \%$ \\
\hline Total & 254 & $100,0 \%$ \\
\hline
\end{tabular}

En lo referente a los niveles de actuación en la educación básica de los encuestados, 27 son profesores en educación infantil (11\%); la mayoría de los 100 encuestados pertenece a escuelas primarias (41,4\%); 52 actúan en el nivel medio (21,7\%); 11 participan en la educación de jóvenes y adultos (4,6\%); 14 actúan en la enseńanza de profesiones (15,3\%); y 37 en otros tipos de educación, como ONG y escuelas rurales $(15,3 \%)$. Cuatro personas $(0,2 \%)$ no respondieron a esta pregunta. Es posible que no estuvieran contratados en el período en el que respondieron al cuestionario, pero se los caracteriza como profesores de educación básica. La Tabla 2 contiene estos datos.

\section{Tabla 2}

Distribución del numero de participantes por nivel de actuación profesional

\begin{tabular}{lcc}
\hline Nivel de actuación & Número & Porcentaje \\
\hline Educación infantil & 27 & $11,0 \%$ \\
\hline Escuela primaria & 100 & $41,4 \%$ \\
\hline Secundaria & 52 & $21,7 \%$ \\
\hline Educación de jóvenes y adultos & 11 & $4,6 \%$ \\
\hline Formación Profesional & 14 & $5,8 \%$ \\
\hline Otros tipos de Enseñanza & 37 & $15,3 \%$ \\
\hline No respondieron & 4 & $0,2 \%$ \\
\hline Total & 245 & $100,0 \%$ \\
\hline
\end{tabular}

En cuanto a la formación de estos profesores participantes, los datos indican que la mayoría, 191 (78\%), tiene formación de alto nivel; $85(34,6 \%)$ curso de especialización; 94 (38,8\%) máster; y 12 (4,8\%) doctorado. Los graduados en cursos de pregrado y profesorado suman 48 (19,7\%); una minoría, 4 $(1,6 \%)$, cuenta con formación de nivel medio. Solo $2(0,6 \%)$ no respondieron. En la Tabla 3 se detallan estos datos.

\section{Tabla 3}

Distribución del número de participantes por grado de formación

\section{Grado de formación Número Porcentaje}

Enseñanza normal de nivel medio

$4 \quad 1,6 \%$

\begin{tabular}{lcc} 
Graduación & 10 & $4,2 \%$ \\
\hline Graduación Profesorados & 38 & $15,5 \%$ \\
\hline Especialización & 85 & $34,6 \%$ \\
\hline Máster & 94 & $38,8 \%$ \\
\hline Doctorado & 12 & $4,8 \%$ \\
\hline No indicado & 2 & $0,6 \%$ \\
\hline Total & 245 & $100,0 \%$ \\
\hline
\end{tabular}

Así, la muestra nos permite indicar que el estudio está compuesto por una serie de respuestas expresivas (245) de los profesores; cubre a profesores de todas las regiones de Brasil; involucra a docentes de diferentes niveles de educación básica: Educación infantil, Escuela primaria, Secundario, Educación de jóvenes y adultos; y Formación profesional y diferentes niveles de formación: Enseñanza normal de nivel medio, Graduación, Graduación Profesorados, Especialización, Máster y Doctorado. 
Instrumento y procedimientos de aplicación y análisis

Los datos fueron recolectados a través de las respuestas de docentes de Brasil en el "Cuestionario sobre Condiciones y Contextos para el Aprendizaje de la Enseñanza”, propuesto por Marcelo (2017). Originalmente, este cuestionario fue propuesto por investigadores de la Universidad de Sevilla. Fue revisado por los autores de este texto para la adecuación de las preguntas al contexto brasileño, pero con pocos cambios con respecto al documento original. Para esta adecuación, los autores, también docentes de educación básica, respondieron el cuestionario.

Se aplicó en la plataforma Google Forms. El cuestionario consta de una parte de identificación de los encuestados, y una segunda parte, de sesenta y cinco preguntas, sobre el aprendizaje docente. El enfoque de las preguntas busca indicar las relaciones con los contextos preferidos para el propio aprendizaje profesional de los docentes en situaciones formales e informales, con el fin de mejorar sus conocimientos profesionales. En la elaboración de las preguntas se distinguieron situaciones que favorecen la expresión de las diferencias que existen entre el profesorado no universitario (en función del nivel escolar y de la experiencia profesional) con relación a los contextos preferidos para su propio aprendizaje profesional (Marcelo, 2017). Las preguntas sobre el aprendizaje contienen ítems en la escala Likert, con cinco medidas que van de mucho a nunca (mucho, a menudo, continuamente, poco, nunca).

También se incluyó el consentimiento informado para cumplir con los estándares éticos en la investigación con seres humanos. Para involucrar al mayor número de participantes, se contactó a profesores de universidades brasileñas, que colaboraron en la difusión del instrumento de investigación ${ }^{2}$, buscando atender como criterio que la muestra contara con la participación de docentes de educación básica de todas las regiones de Brasil.

Después de las lecturas y los análisis de las respuestas, considerando las recomendaciones de Gatti
\& André (2010), se agruparon y categorizaron los datos obtenidos. Primero, se hizo la caracterización de los profesores participantes y, luego, se categorizaron las respuestas. Para este artículo consideramos del informe de investigación las respuestas referidas a las alternativas "continuamente" y "mucho", lo que resultó en la categorización de cuatro categorías de aprendizaje docente: aprendizaje en la práctica, aprendizaje mediante investigación, aprendizaje interactivo en grupos colaborativos, aprendizaje mediante autoformación.

Para la composición de cada una de las categorías se infirieron las dimensiones de enseñanza aprendizaje expresadas por los docentes en las respuestas, las cuales fueron organizadas en tablas presentadas en los resultados, cuya elaboración es propia de los autores.

\section{Resultados}

\section{Dimensiones del aprendizaje de la docencia de los profesores de educación básica}

En el análisis de las respuestas de los docentes al cuestionario de investigación, se entienden como categorías constitutivas las siguientes dimensiones del aprendizaje docente: en la práctica docente, a través de la investigación, en prácticas de enseñanza colectiva, a través de la autoformación. La categorización considera autores que ayudan a explicar y comprender la constitución del aprendizaje y las relaciones con la formación y profesionalización docente, en una sociedad conectada a los más diversos recursos tecnológicos de información y comunicación.

Los resultados se organizan leyendo las respuestas a las preguntas del cuestionario. Para ayudar a comprender estas respuestas, las tablas siguientes contienen las dimensiones de las respuestas en cada una de las categorías y las expresiones de los participantes de la investigación. Los participantes se indican con la letra P. porque se refieren a profesores y están numerados según el orden en que aparecen las respuestas en el informe de investigación. 


\section{Tabla 4}

Aprender en la práctica docente

\section{Dimensiones}

Los profesores aprenden cuándo:

\section{Las respuestas del profesor}

Adaptan la forma de enseñar a las especificidades del contenido y a la necesidad de los estudiantes.
"La profesión de profesor es una lucha constante por aprender. Aprendemos de cada detalle de nuestro día a día y, cuando estamos dentro de esta profesión, todo lo que sucede fuera podemos relacionarlo con las asignaturas con las que trabajamos y la forma en que enseñamos". [P.42]

"Aprendo cuando adapto mi forma de enseñar a las necesidades específicas delos estudiantes”. [P. 63]
Crean sus propios materiales didácticos para sus clases.
"Aprendo cuando creo mis propios materiales didácticos para mis clases". [P. 105]
Buscan cambiar sus propias experiencias con nuevas estrategias de enseñanza.
"Apréndalo observando y arriesgando nuevas experiencias de enseñanza y aprendizaje de forma autónoma”. [P. 37]

"Aprendo cuando utilizo materiales de otros compañeros profesores en mis propias clases". [P. 194]

"Aprendo observando cómo mis alumnos aprenden e interactúan con las metodologías que propongo". [ P. 162]

En las interacciones con los estudiantes. "Aprendo de mis alumnos cuando me dicen cómo enseño”. [P. 85]

"Aprendo cuando interactúo con los estudiantes en la escuela, fuera del ambiente del aula”. [P. 59]

Informan la manera en que enseñan a sus alumnos y a otras personas.

"Aprendo cuando describo a otras personas, casos o situaciones, que he experimentado en mi práctica docente”. [P. 28]

"Aprendo cuando reflexiono sobre mis propias creencias y concepciones acerca de la enseñanza”. [P. 220]

Reflexionan sobre sus propias creencias y concepciones sobre la enseńanza.

"Aprendo cuando reflexiono sobre mi propia práctica”. [P. 13]

"Aprendo cuando reflexiono sobre mis fortalezas y debilidades como maestro". [P. 132]

"Aprendo cuando analizo las reacciones de los estudiantes en clase". [P. 63]

Analizan las reacciones de los estudiantes en la clase.

"Aprendo cuando me doy cuenta de que hay contradicciones entre

Examinan sus ideas sobre la enseñanza y cómo las ponen en práctica, indicando las contradicciones existentes. mis ideas sobre la enseñanza y cómo las coloqué en la práctica de la enseñanza”. [P. 21]

Evalúan las tareas de aprendizaje de los "Aprendo cuando evalúo las tareas de aprendizaje de los estudiantes". [P. 08] alumnos.

"Aprendo, analizo y entiendo el porqué de las respuestas equivocadas de mis alumnos a las preguntas que formulo". [P. 114] 


\section{Tabla 5}

\section{Aprender a través de la investigación}

\section{Dimensiones}

Los profesores aprenden cuándo:

\begin{tabular}{|c|c|}
\hline \multirow{4}{*}{$\begin{array}{l}\text { Participo en grupos de } \\
\text { investigación. }\end{array}$} & $\begin{array}{l}\text { "Aprendo participando en un grupo de investigación específico en el área de formación de } \\
\text { profesores". [P. 132] }\end{array}$ \\
\hline & "Aprendo cuando investigo y discuto con mis colegas colectivamente". [P. 97] \\
\hline & "Participando en un grupo de investigación dentro de la institución o en universidades". [P. 76] \\
\hline & "Aprendo asistiendo a seminarios, congresos, talleres". [P. 29] \\
\hline \multirow{2}{*}{$\begin{array}{l}\text { Investigo por mi propia } \\
\text { iniciativa. }\end{array}$} & "Investigando en libros sobre educación”. [P. 228]. \\
\hline & "Aprendo, fundamentalmente, investigando: dar clases". [P. 38] \\
\hline \multirow{4}{*}{$\begin{array}{l}\text { Busco conocimiento en } \\
\text { situaciones informales. }\end{array}$} & $\begin{array}{l}\text { "Aprendo cuando visito exposiciones de arte, museos, cuando conozco otros lugares en mi } \\
\text { propio país o región u otros países, obteniendo más conocimiento sobre la cultura de la sociedad, } \\
\text { y sobre el contexto en el que vive la gente, aprendo participando en manifestaciones culturales y } \\
\text { políticas". [P. 12] }\end{array}$ \\
\hline & $\begin{array}{l}\text { "Aprendo haciendo cosas, como artesanías, proponiendo actividades productivas (en el sentido } \\
\text { manual), para los alumnos". [P.139]. }\end{array}$ \\
\hline & $\begin{array}{l}\text { "Aprendo leyendo novelas y viendo películas de ciencia ficción, especialmente aquellas que } \\
\text { abordan distopias". [P. 56] }\end{array}$ \\
\hline & "Aprendo visitando librerías y bibliotecas". [P. 111] \\
\hline
\end{tabular}

\section{Tabla 6}

Aprendizaje en prácticas docentes colectivas

\section{Dimensiones}

Los profesores aprenden cuándo:

\section{Las respuestas del profesor}

"Compartir con compañeros docentes y los problemas que surgen en la vida cotidiana de la escuela". [P. 105]

Se produce la

"Planificar clases en colaboración con los docentes de la escuela”. [P. 97]

colaboración entre

"Reuniones de los consejos de coordinación en la escuela". [P.18]

sujetos.

"Aprendo cuando investigo y hablo con mis colegas en conjunto". [P. 124]

"Aprendo de los comentarios de los estudiantes sobre el desarrollo de las clases, el programa de enseñanza, las evaluaciones”. [P. 176]

\footnotetext{
Hay una confluencia en diferentes contextos, formales e informales, de experiencias, conocimientos y situaciones.

"Aprendo en las interacciones que establezco en el ambiente educativo, con lecturas de hechos y eventos cotidianos que requieren actitudes rápidas y precisas". [P. 162]

"Aprendo cuando converso con profesores en situaciones informales". [P. 27]

"Intercambiar ideas y puntos de vista sobre la educación con otros docentes". [ P. 58]

“Comparto experiencias sobre mi práctica”. [P. 234]

"Uso materiales de otros compañeros en mis propias clases". [P. 119]

Se producen relaciones "La mejor forma que me ha proporcionado el aprendizaje en la docencia es el intercambio con solidarias. otros profesionales de la escuela, como profesores y coordinadores. A veces es muy complicado, hay profesores a los que no les gusta escuchar dificultades o informar de su práctica, pero cuando encontramos profesionales dispuestos a ayudar, es un apoyo importante en la práctica, sobre todo ayudando a superar dificultades, y así, adquiriendo nuevos conocimientos". [P. 58]
} 
Tabla 7

Aprender mediante autoformación

\section{Dimensiones}

Los profesores aprenden cuándo:

\section{Las respuestas del profesor}

Realizan la reflexión.

"Aprendo cuando dejo mi zona de confort y quiero mejorar". [P. 39]

Realizan estudios individuales para ampliar su aprendizaje.

“Básicamente, aprendo investigando: para dar clases”. [P.29]

Buscan acciones formativas que ofrecen las instituciones.

"Siempre aprendo cuando busco la autoeducación, porque los sistemas educativos ofrecen poca educación continua para los docentes". [P 126]

Examinan sus ideas sobre la enseñanza y cómo las ponen en práctica, indicando las contradicciones existentes.

"Aprendo cuando me doy cuenta de que hay contradicciones entre mis ideas sobre la enseñanza y cómo las coloqué en la práctica de la enseñanza”. [P. 21]

Hacen narrativas para otros.

"Cuando le hablo a mi familia sobre mis prácticas y ellos dan ideas y opiniones sobre mi práctica”. [P.75]

\section{Conclusiones y discusiones}

\section{Dimensiones del aprendizaje de la docencia de los profesores de educación básica}

En las conclusiones y discusiones se consideran las dimensiones expresadas en las respuestas de los docentes en comparación con las referencias de diferentes autores que discuten y analizan el aprendizaje docente. Los datos de la encuesta nos permitieron categorizar en: aprendizaje en la práctica, aprender a través de la investigación, aprendizaje interactivo en grupos colaborativos, aprendizaje a través de la autoformación, como ya se ha indicado. Así se presentan las conclusiones y discusiones en ese orden.

\section{Aprender en la práctica docente}

Este estudio sostiene que el aprendizaje en la práctica no se trata de un aprendizaje tecnicista y reducido a un saber hacer, tal y como indica Zeichner (2008). Los datos recogidos en la investigación, descritos en la Tabla 4, indican que los profesores reconocieron como situaciones de aprendizaje en la propia práctica cuando: adaptan la forma de enseñar a las especificidades del contenido y necesidad de los estudiantes, crean sus propios materiales didácticos para sus clases, buscan cambiar sus propias experiencias con nuevas estrategias de enseñanza. Martins (2016) concibe al aprendizaje en la práctica basado en la teoría como expresión de la práctica y se apoya en la comprensión de la experiencia de una determinada práctica pedagógica forjada por el profesor para enfrentar a los problemas impuestos por la práctica.

Aprender, según los maestros, implica analizar las reacciones de los estudiantes en la clase cuando informan cómo enseñan, evaluar las tareas de aprendizaje de los estudiantes y entender el porqué de las respuestas incorrectas de sus estudiantes, ante las preguntas hechas por los profesores. Al describir el aprendizaje de los docentes con la relación a los estudiantes, Vailllant \& Marcelo (2016) se refieren a la gestión del aprendizaje como una de estas dimensiones. Así, si pretendemos una práctica pedagógica articulada con los intereses de las clases populares, aprender con ellas cómo generar una pedagogía es fundamental (Martins, 2009).

En la categoría de Aprendizaje en la práctica docente, Oliveira-Formosinho (2016) defiende la formación en contexto centrada en las prácticas y el 
compromiso del docente desde la fase de relevamiento de las necesidades hasta la planificación, ejecución y evaluación de su formación. Desde este punto de vista, los profesores aprenden reflexionando sobre sus propias creencias y concepciones sobre la enseñanza, examinando sus ideas sobre la enseñanza y cómo las ponen en práctica, indicando las contradicciones existentes y utilizando nuevos materiales didácticos. Las respuestas de los docentes resaltan la importancia de registrar, reflexionar y analizar si los procedimientos utilizados fueron adecuados. Al evaluar estas situaciones comprenden sus avances y necesidades como docentes. Es a partir de este sistema reflexivo que el aprendizaje en la práctica es una posibilidad.

En este estudio, se entiende como situaciones de aprendizaje de los docentes en la propia práctica cuando estos relatan la forma en que enseñan a sus estudiantes y a otras personas, reflexionan sobre sus propias creencias y concepciones sobre la enseñanza, analizan las reacciones de los estudiantes en la clase, examinan sus ideas sobre la enseñanza y cómo las ponen en práctica, indicando las contradicciones existentes, buscan cambiar sus propias experiencias con nuevas estrategias de enseñanza, informan la forma en que enseñan a sus alumnos y a otras personas, evalúan las tareas de aprendizaje de los alumnos, utilizan nuevos materiales didácticos. Se trata de situaciones de aprendizaje formal e informal, aunque en su mayoría tienen lugar en el espacio escolar.

Desde una perspectiva marxista, la transformación del mundo tiene lugar a través del trabajo productivo, y que, al producir, los hombres se producen a sí mismos en aquello que producen. Este principio se expresa en la tesis XI sobre Feuerbach: "Los filósofos se han limitado a interpretar el mundo de diferentes maneras; pero lo que importa es transformarlo" (Marx \& Engels, 2005, p. 118). Cabe destacar que Gramsci (2000), a partir de esta tesis, entiende que el proceso de transformación social ocurre orgánicamente, tomando como referencia el proceso de producción y socialización del conocimiento por parte de un grupo social especializado, dentro de su categoría de trabajadores de la educación. Al respecto, es importante tener en cuenta que es deseable que los profesores se constituyan como intelectuales orgánicos, al planificar, realizar y evaluar su trabajo pedagógico en determinadas condiciones y relaciones sociales. Sin embargo, en el contexto social actual, de una pedagogía de resultados se aprecia más el cumplimiento de metas, en las que los docentes se convierten en ejecutores de las determinaciones de los sistemas educativos.

\section{Aprender a través de la investigación}

El aprendizaje de la docencia a través de la investigación forma parte de un movimiento desarrollado desde finales del siglo XIX con las propuestas de Dewey (1959) sobre la enseñanza y el aprendizaje a partir de la experimentación al "investigar la idoneidad, el valor de cualquier índice en particular; cuando experimentamos verificamos su validez y sabemos cuál es la garantía de que los datos existentes realmente indican la idea sugerida, para que justifique aceptarla” (p. 21).

La inserción de la investigación en los procesos de aprendizaje docente toma protagonismo en las décadas de 1960 y 1970, durante una reforma curricular del sistema educativo inglés con Lawrence Stenhouse (1975), quien propuso a los docentes llevar a cabo investigaciones, como una forma de mejorar el proceso de enseñanza y aprendizaje. Esta proposición es tomada por Lüdke (2012) con la expectativa de caracterizar al docente como un intelectual crítico, capaz de reflexionar sobre su actividad profesional y sobre la realidad social como docente investigador.

Para Darling-Hammond \& Richardson (2009) la investigación expresa un nuevo enfoque en los procesos de formación docente. Entender al profesor como sujeto activo de su aprendizaje es un cambio poderoso, al "hacer al profesor productor de conocimiento y no solo consumidor" (Romanowski, 2010, p. 115).

El movimiento de aprendizaje a través de la investigación ha sido analizado en los últimos años por diferentes investigadores: André (2019), que defiende a la investigación como principio didáctico en la formación de profesores; Lüdke (2012) argumenta que los docentes son investigadores; Martins (2016) desarrolla la perspectiva de la formación docente a través de la investigación-enseñanza; Fiorentini \& Crecci (2016) buscan comprender el aprendizaje y el 
desarrollo profesional de los docentes en comunidades de investigación. La comunidad de investigación es un proceso formativo valorado por CochranSmith (2012) al considerar el aprendizaje docente en la interacción entre la investigación y la práctica formativa de profesores. Estas posibilidades se expresan en la Tabla 5.

Se entiende que, en el proceso de aprendizaje a través de la investigación, el docente busca superar las prácticas marcadas por la aplicación del conocimiento para convertirse en un indagador atento de la práctica, como un proceso abierto de nuevas posibilidades. Esta disposición y propensión a realizar un saber hacer mediado permanentemente por la indagación y la reflexión crítica permite la autonomía del docente a través del proceso de toma de decisiones conscientes.

El movimiento provocado por la acción inves/ tigadora posibilita el desarrollo de la autonomía, al concebir al docente como sujeto que analiza de manera sistemática los hechos que envuelven el contexto de la práctica, examinando incertidumbres, considerando conflictos, situaciones de diversidad y diferentes experiencias sociales.

Dice André (2016) que bajo la perspectiva del aprendizaje a través de la investigación el profesor se convierte en sujeto de sus propios ideales, porque piensa por sí mismo, siendo capaz de "elegir entre alternativas, decidir el camino a seguir, implementar acciones y tener argumentos para defender sus elecciones y sus acciones" (p. 20). Las dimensiones indicadas por los docentes indican su aprendizaje a través de la investigación cuando participan en grupos de investigación e investigan por su propia iniciativa.

En este sentido, el espectro de alternativas a ser exploradas por los docentes es amplio, posibilitando una gran diversidad de acciones individuales $y$ colectivas, como se identificó en el cuestionario de investigación "¡cómo aprenden los docentes en una sociedad conectada?" y abarca iniciativas en el marco institucional formal y ámbitos no institucionalizados o informales. En el contexto institucional formal, observamos la inserción de actividades de investigación en los cursos de formación inicial como principio formativo (Fontana, 2016).
En una tercera dimensión de respuestas a la encuesta, fueron descritas las posibilidades en el contexto no institucionalizado e informal. En este conjunto de respuestas aparecen aspectos informales y subjetivos del aprendizaje a través de la investigación, que van desde las demandas de intereses personales como forma de preparación para el trabajo docente, hasta actividades más libres y desinteresadas, como viajes, visitas a exposiciones artísticas, bibliotecas culturales, producción de artesanías, lecturas, cine y otros.

\section{Aprendizaje en prácticas docentes colectivas}

Veiga (2008) destaca que la organización del proceso didáctico en la perspectiva colaborativa será la que asegure el alcance y la eficacia de la enseñanza. Los aprendizajes colectivos presuponen la colaboración entre sujetos y han sido centrales en la discusión de varios investigadores, que lo entienden todavía como un desafío, ya que aún predomina la práctica individual por sobre los intentos de prácticas colectivas auténticas (Roldão, 2007).

Para la autora, la colaboración es el "cemento organizacional”, es esencial para el desarrollo eficaz de proyectos escolares (Roldão, 2007, p. 89). Lima \& Fialho (2015) argumentan que la colaboración entre profesores es un factor poderoso de aprendizaje profesional y de eficacia para las escuelas. Ambos dialogan con Cartaxo et al. (2020), que presentan el potencial del proceso formativo con profesores en la enseńanza fundamental, a partir de las propias prácticas en el contexto de trabajo escolar.

Para Aubert et al. (2018) este aprendizaje se caracteriza por la interacción y comunicación producida en comunidades de aprendizaje, con diálogos igualitarios, en interacciones que potencian el aprendizaje instrumental, los aprendizajes significativos con sentido personal y social y la apreciación de la solidaridad, la igualdad y las diferencias. Investigaciones y teorías de referencia para el aprendizaje dialógico se desarrollan y han tenido éxito en superar el fracaso escolar y mejorar la convivencia en las llamadas comunidades de aprendizaje en España, Brasil y Chile (Aubert et al., 2018). 
Para Vaillant \& Marcelo (2012) este es un modelo con una visión más compleja y poderosa para el proceso de aprendizaje y enseñanza, ya que se entiende que hay una confluencia en diferentes contextos, formales e informales, de experiencias, conocimientosy situaciones. Espacios que promueven la interacción entre los sujetos y la indagación de manera sistemática y crítica sobre la enseñanza, el aprendizaje y la organización escolar. Es necesario crear relaciones de colaboración que superen la hegemonía de las propuestas educativas individualistas, basarse en principios de reciprocidad e intercambio, ensayar nuevas prácticas, promover la indagación sistemática y atender a la diversidad de sujetos.

Los docentes participantes en este estudio expresaron que aprenden intensamente en los espacios escolares por las interacciones y prácticas colectivas (Tabla 6): se produce la colaboración entre sujetos, hay una confluencia en diferentes contextos, formales e informales, de experiencias, conocimientos y situaciones, se producen relaciones solidarias.

Hargreaves \& O’Connor (2018) y Nóvoa (2019) postulan lo colectivo, en un proceso de interacción de experiencias que favorecen la mejora e innovación de la profesión y el trabajo docente.

Las búsquedas y consultas en Internet están cada vez más presentes y ganaron mayor visibilidad durante la pandemia causada por el Covid 19, como señala la investigación de Romanowski (2020). Los profesores comentan que aprenden a través de las búsquedas de contenidos y recursos para las clases en Internet y redes sociales, con lecturas sobre educación en revistas, en videos de profesores, con la consulta de sitios web, blogs, etc. de docentes, con la participación en cursos y especializaciones en la modalidad a distancia; por tanto, una intensificación del aprendizaje desde la perspectiva de una sociedad conectada.

El aprendizaje no planificado y en diferentes situaciones y espacios de la escuela implica escuchar ideas y propuestas de otras personas no relacionadas con el mundo de la educación, la participación en redes o grupos de docentes fuera de la escuela (sindicato, redes de docentes, etc.), colaboraciones con organizaciones no gubernamentales que desarrollan proyectos de solidaridad, visitas a organizaciones, empresas e instituciones, aunque estos aprendizajes pueden ser menos notados por los maestros.

\section{Aprender mediante autoformación}

El aprendizaje por autoformación es un proceso que ocupa espacio en las discusiones sobre la formación, porque se considera que las personas, individualmente y en grupo, definen sus itinerarios de aprendizaje destacando las experiencias relevantes para el desarrollo profesional y personal. Según Pineau (1988), las personas asumen responsabilidades por el proceso formativo y desarrollan un trayecto de autoeducación de manera permanente, dentro de un contexto, con un nivel deseable de autonomía. Pineau \& Breton (2021) explican que "una teoría de la formación permanente" ocurre "en dos etapas -experiencial y formal-y en tres movimientos: auto, socio, y eco formación" (p. 13) y así la formación involucra la dimensión personal, articulada con lo social.

Para Bragança (2011), "la autoeducación es la dimensión personal del reencuentro reflexivo en el que las cuestiones del presente nos llevan a problematizar el pasado y construir un proyecto sobre el futuro" (p. 159). Los datos de la investigación indican que los docentes realizan estudios individuales para ampliar su aprendizaje a través de la lectura de artículos de revistas educativas, textos sobre el contenido de la asignatura impartida, manuales y libros sobre educación. Para Guimarães \& Faria-Fortecoëf (2021) las narrativas de los profesores permiten aprendizajes que favorecen a la constitución de los saberes docentes.

El proceso de autoformación no tiene un programa cerrado, se describe como una formación informal, que tiene como espacio privilegiado la experiencia (personal y profesional) y la reflexión sobre este proceso, considerando que en el aprendizaje de adultos existe necesidad de autonomía y flexibilidad respecto de los modelos escolares conocidos. En el contexto de las investigaciones sobre la autoeducación, Pineau señala que este es un proceso de interacción cognitiva de cada persona que, individualmente o en grupo, reúne y 
ordena los diferentes momentos de la vida, construyendo un tiempo singular y con consistencia temporal, que le permite tomar conciencia de sí mismo y del otro, conocer y valorar el trayecto formativo considerando las experiencias en determinados momentos, valorar su propia existencia. Se trata de "vivir la propia experiencia" constituida a lo largo de su historia de vida personal y profesional (Hoça \& Romanowski, 2018). Las respuestas que indican que los docentes estudian fueron muchas y la búsqueda por actualización de los conocimientos sobre los que los profesores dan clases es evidente, en la lectura de artículos científicos, libros especializados, psicopedagogía, sobre las fases del desarrollo cognitivo y las formas de aprendizaje humano, filosofía, donde buscan ampliar su visión y comprensión en varias áreas. Este aprendizaje se destacó en las respuestas abiertas, descritas en la Tabla 7.

En el aprendizaje por autoformación, Vaillant \& Marcelo (2012) destacan la existencia de investigaciones realizadas con tres enfoques predominantes: el psicológico, concebido por Pineu a partir de las referencias de desarrollo, crecimiento personal y profesional; el sociológico, desde la perspectiva de Dumazedier, que destaca la autoformación como una necesidad social, y el pedagógico, de Berliner, que entendió la necesidad de transmitir los resultados de la investigación a la formación. El aprendizaje por autoformación depende de una inversión que cada profesional realiza, ante el abanico de acciones formativas que ofrecen las instituciones, para acceder a diversas publicaciones, artículos y libros impresos y digitales, y a redes sociales. Sin embargo, la educación continua no siempre es ofrecida por los sistemas educativos, como dicen los profesores.

La intensidad con la que se comparten experiencias y conocimientos con personas de todo el mundo, a partir de los contactos generados en diferentes redes, saca a relucir aprendizajes considerados significativos para el proyecto personal y profesional de los docentes. Como afirman Vaillant \&Marcelo (2012), no se trata de una moda educativa, no se limita a la lectura y la escritura, sino que es un movimiento de aprendizaje largo y complejo.

\section{Consideraciones finales}

El artículo puso en discusión los procesos de aprendizaje de la docencia de profesores de educación básica en el siglo xxi en una sociedad conectada, con el objetivo de conocer cómo son desarrollados los procesos de aprendizaje de los docentes, en alternativas formales e informales, para mejorar su conocimiento profesional.

Las sistematizaciones realizadas expresan que los profesores están comprometidos con su formación y buscan diferentes formas y modos de mejorar sus conocimientos y, sobre todo, llevar a cabo su práctica con compromiso. A partir de esto reafirmamos el "supuesto de que las sistematizaciones teóricas de los diferentes enfoques sobre las prácticas de formación docente se producen socialmente a partir de determinadas circunstancias históricas y que tales circunstancias determinan los tipos de relaciones sociales que forjarán las tecnologías utilizadas en la relación pedagógica”, como propone Martins \& Romanowski (2010, p. 205).

El proceso colectivo de desarrollo de la investigación intergrupal es un punto fundamental para favorecer el intercambio entre investigadores y áreas de conocimiento desde la perspectiva de la interdisciplinariedad, con el fin de ampliar la colaboración científica, profundizando la discusión y el análisis de los temas y problemas investigados. Esta práctica de investigación, en conjunto, favorece una nueva cultura académica, superando el individualismo y avanzando hacia prácticas más colaborativas (Lima $\&$ Fialho, 2015). Los profesores destacaron esta posibilidad de aprendizaje en sus respuestas y, como señala Veiga (2008), en la búsqueda de la mejora del proceso docente. Se pone de manifiesto que la colaboración es intensa en el espacio escolar, como postula Nóvoa (2019), quien afirma que "no es posible aprender la profesión docente sin la presencia, el apoyo y la colaboración de otros docentes", realizando experiencias que involucran la planificación llevada a cabo colectivamente. De hecho, las colocaciones de los encuestados nos permiten afirmar que existen 
relaciones colaborativas para propuestas educativas basadas en principios de reciprocidad e intercambio, como recomiendan Vaillant \& Marcelo (2012), que son embrionarias para probar nuevas prácticas, indagación sistemática y diversidad de sujetos. Sin embargo, aún no se materializan como comunidades de práctica, como proponen Aubert et al. (2018). Los profesores están llevando a cabo cada vez más la transición del aislamiento, de la individualización hacia el fortalecimiento de los colectivos docentes que constituyen comunidades, pero, aunque los docentes continúan investigando y estudiando para mejorar su profesionalización, la interacción de los docentes en las redes sociales es menos frecuente. Se observa que en el contexto de la pandemia del Covid 19 se intensificaron las interacciones a través de la web (Romanowski et al., 2020). Se necesitan más investigaciones para examinar este nuevo escenario de aprendizaje docente en una sociedad conectada.

Los indicadores de las contribuciones de la formación informal expresan una mayor valoración de la propia práctica docente, como una contribución en el proceso de formación y profesionalización docente. Como destacan varios autores y los docentes encuestados, este aprendizaje en la práctica genera nuevas posibilidades de enseñanza, conocimiento y nuevos aprendizajes para profesores, una contribución de la actuación docente, en contraste con la realización de la acción misma, sin la debida reflexión, en la que la formación prescinde de la reflexión, como señalan Diniz-Pereira \& Zeichner (2017). Es al reflexionar sobre la acción que es posible entender esta acción, que hay praxis.

El aprendizaje a través de la investigación es más intenso cuando parte de la búsqueda y lectura de materiales con los que se trabaja cotidianamente en el proceso de enseñanza y aprendizaje. En los registros de los docentes se valora este esfuerzo, que instiga a mejorar su educación mediante iniciativas de autoformación, más ligadas a la búsqueda de alternativas para mejorar la práctica docente que a la investigación, por lo que no se trata de convertirse en profesor investigador como proponen Ludke (2012) y Cochran-Smith (2012). La investigación se realiza más por consulta que por participación en un grupo de investigación, desde la perspectiva de Fiorentini \& Crecci (2016), y por aprendizaje, considerando la investigación como principio formativo (André, 2019). Sin embargo, la investigación realizada apunta a que raras veces los profesores realizan una investigación sistemática. Los profesores realizan consultas más puntuales e informales.

La autoformación aparece atravesando el aprendizaje en la práctica, en la investigación y en lo colaborativo, porque corroborando con Pineau (1988) y Pineau \& Breton (2021), los docentes asumen responsabilidades parasu formación medianteconsultas, conversaciones, estudios y reflexiones sobre la práctica, pero los datos se limitan a indicar la consumación de la autonomía docente. La orientación del aprendizaje hacia la autonomía pedagógica es más evidente en las respuestas de los profesores Vaillant \& Marcelo (2012), pero se necesitan nuevas investigaciones que permitan indicaciones con este enfoque del aprendizaje docente.

Es posibleafirmarque, en el contextoactual, el proceso de formación docente se amplía, con más posibilidades en educación formal, con la oferta intensificada de cursos, conferencias y eventos, que se enriquece con los que ofrece la formación informal. Con el aumento del acceso a nuevas fuentes de conocimiento proporcionadas por Internet, por impulso de la formación colectiva, la formación informal y formal pauta nuevas perspectivas de profesionalización docente. Paradójicamente, esa expansión, al acercar contribuciones, plantea demandas de una mejor profesionalización de los docentes, que esta investigación deja abiertas como temas a ser indagados. Estos aprendizajes, asociados a su formación en la perspectiva del desarrollo profesional, contribuyen a mejorar la práctica docente, favorecen los procesos de reflexión y amplían las interacciones y la participación de los docentes en la escuela; en particular, expresan un cambio en la relación de los docentes con sus alumnos. Todas estas relaciones se llevan a cabo horizontalmente y son colaborativas. Hoy, la escuela se destaca como un espacio donde la formación docente acontece en el aprendizaje formal e informal. 


\section{Referencias}

André, M. (2002). Formação de professores no Brasil (1990-1998). MEC/Inep/Comped. http://portal. inep.gov.br/documents/

André, M. (2019). Pesquisa, formação e prática docente. In M. André (Org.), O papel da pesquisa na formação e na prática dos professores (6a ed., pp. 55-69). Papirus.

Aubert, A., Flecha, L. A., García, C., Flecha, R., \& Racioneiro, A. S. (2018). A aprendizagem dialógica na sociedade da informação. EdUFSCar.

Brasil. (1996). Lei no 9.394/96. Presidência da República. Casa Civil. (Estabelece as diretrizes da educação nacional). http://www.planalto. gov.br/ccivil_03/leis/19394.htm

Brzezinski, I., \& Garrido, E. (2006). Formação de profissionais da educação (1997-2002). MEC/ Inep. http://inep.gov.br/documents/186968/ 484330/

Brzezinski, I. (2014). Formação de profissionais da educação (2003-2010). MEC/Inep. http://inep. gov.br/documents/186968/484330/

Bragança, I. (2011). Sobre o conceito de formação na abordagem (auto)biográfica. Educação, 34(2), 157-164. https://revistaseletronicas.pucrs.br/ ojs/index.php/faced/article/view/8700

Cartaxo, S., \& Romanowski, J. P. (2019). Research on teacher education in Brasil. Proceedings of the 19 Biennial Conference of Internacional Study. Association on Teachers and Teachin.

Cartaxo, S., Mira, M., \& Gasparim, R. (2020). Formação de professores da educação básica: análise dos processos formativos. Revista $d a$ FAEEBA-Educação e Contemporaneidade, 29(57), 78-94. https://doi.org.10.21879/ faeeba2358-0194.2020.v29.n57.p78-94

Castells, M. (2020). O digital é o novo normal. Fronteiras do pensamento. https://www.fronteiras. com/artigos/o-digital-e-o-novo-normal

Cochran-Smith, M. (2012). Aprender a ensinar nunca termina? Kappa Delta Pi Record, 47(1), 22-24. https://doi.org/10.1080/00228958.20 11.10516719
Darling-Hammond, L., \& Richardson, N. (2009). Teacher learning: What matters? Educational leadership, 66(5), 46-53. https://www.ascd.org/ $\mathrm{el} /$ articles/teacher-learning-what-matters

Darling-Hammond, M., Wei, R. C., \& Johnson, C. M. (2009). Handbook of Education Policy Research. Taylor \& Francis.

Dewey, J. (1959). Democracia e Educação: Introduçâo à filosofia da Educação. Nacional.

Diniz-Pereira, J., \& Zeichner, K. M. (2017). A pesquisa na formação e no trabalho docente. Autêntica.

Fiorentini, D., \& Crecci, V. (2016). Interlocuçôes com Marilyn Cochran-Smith sobre aprendizagem e pesquisado professor em comunidades investigativas. Revista Brasileira de Educação, 21, 505-524. https://doi.org/10.1590/S141324782016216526

Fontana, M. I. (2016). A Pesquisa em Educação do Campo no curso de pedagogia: contribuiçóes do programa observatório da educação. Cadernos de Pesquisa: Pensamento Educacional, 11 (especial), 239-257. https://seer.utp.br/index.php/a/ article/view/1461/1234

Freire, P. (1996). Pedagogia da autonomia. Paz e Terra. Gatti, B., \& André, M. (2010). A relevância dos métodos de pesquisa qualitativa em Educação no Brasil. In W. Weller, \& N. Pfaff (Org.), Metodologias da pesquisa qualitativa em educação: teoria e prática (pp. 29-52). Vozes.

Gramsci, A. (2000). Cadernos do Cárcere (2). Civilização Brasileira.

Gohn, M. (2006). Educação não-formal, participação da sociedade civil e estruturas colegiadas nas escolas. Ensaio: Avaliação e Politicas Públicas em Educação, 14(50), 27-38. https://doi. org/10.1590/S0104-40362006000100003

Guimarães, P., \& Faria-Fortecoëf, C. (2021). A complementaridade entre educação (formal, não formal e informal) e (auto, hetero e eco) formação: uma discussão a partir de autobiografias. Educação (UFSM), 46(1), e32/ 1-22. https://doi. org/10.5902/1984644461311

Hargreaves, A. \& O’ Connor, M. T. (2018). Collabotive profissonalism: when teaching together means learning for all. Corwin. 
Hoça, L. \& Romanowski, J. (2018) Desenvolvimento profissional de professoras alfabetizadoras: malha e laços. Práxis Educativa, 13(2), 481-498. https:// doi.org/10.5212/PraxEduc.v.13i2.0013

Hargreaves, A. (2003). O ensino na sociedade do conhecimento: a educação na era da insegurança. Porto Editora.

Lima, J., \& Fialho, A. (2015). Colaboração entre professores e percepçóes da eficácia da escola e da dificuldade do trabalho docente. Revista Portuguesa de Pedagogia, 49(2), 27-53. http:// hdl.handle.net/10400.3/4370

Lüdke, M. (2012). Desafios para a pesquisa em formação de professores. Revista Diálogo Educacional, 12(37), 629-646. http://dx.doi.org/10.7213/ dialogo.educ.7195

Marcelo, C. (2009). A identidade docente: constantes e desafios. Formaçâo Docente - Revista Brasileira de Pesquisa sobre Formação de Professores, 1(1), 109-131. http://formacaodocente.autenticaedi tora.com.br

Marcelo, C. (2017). ¿Cómo aprenden losprofesores en una sociedad conectada? Proyecto de investigación. https://investigacion.us.es/sisius/sis_proyecto. php?idproy $=29549$

Martins, P. (2009). A didática e as contradiçóes da prática. Papirus.

Martins, P., \& Romanowski, J. (2010). A didática na Formação Pedagógica de Professores. Revista Educação - PUCRS, 33(3), 205-212.

Martins, P. (2016). Pesquisa-ensino na formação de professores e a interlocução com a Educação Básica: princípios e metodologia. In J. Romanowski, P. L. O. Martins, \& S. R. M. Cartaxo, (Org.). Prática de Formação de Professores (pp. 1-20). Champagnat.

Nóvoa, A. (2009). Professores: imagens do futuro presente. Educa.

Marx, K., \& Engels, F. (2005). A ideologia alemã: Feuerbach - a contraposição entre as cosmovisóes materialista e idealista. Martin Claret.

Oliveira-Formosinho, J. (2016). A formação em contexto: a mediação do desenvolvimento profissional praxiológico. In. V. A., Cancian;
S. F. S. Gallina \& N. Weschnfelder, Pedagogias das infâncias, crianças e docências na educação infantil (pp. 87-111). Ipê Amarelo.

Palhares, J. A. (2009). Reflexões sobre o não-escolar na escola e para além dela. Revista Portuguesa de Educação, 22(2), 53-84. https://doi.org/10. 21814/rpe. 13966

Pineau, G. (1988). A autoformação no decurso da vida: entre hetero e a ecoformação. In A. Nóvoa, \& M. Finger (Org.). O método (auto)biográfico e a formação (pp. 65-77). Ministério da Saúde.

Pineau, G., \& Breton, H. (2021). Conquérir son temps par la formation des rythmes de sa vie. Educar em Revista, 37. https://revistas.ufpr.br/ educar/article/view/77919

Romanowski, J. (2010). Formação e profissionalização docente ( $4^{\mathrm{a}} \mathrm{ed}$.). IBPEX.

Romanowski, J. (2018). Tendências da pesquisa em formaçáo de professores: entre o local e o universal. Cadernos de Pesquisa, 25, 207-224, http://dx.doi. org/10.18764/2178-2229.v25n4p207-224

Romanowski, J. (2020). Práticas pedagógicas: inserção das tecnologias na promoção da aprendizagem. In J. P. Romanowski, et al. (Org.), Educação e tecnologias: desafios dos cenários de aprendizagem. Editora Bagai.

Roldáo, M. C. (2007). Colaborar e preciso: Questóes de qualidade e eficácia no trabalho dos professores. Noesis, 71, 2429.

Stenhouse, L. (1975). An introduction to curriculum research and development. Heinemann.

Vaillant, D., \& Marcelo, C. (2012). Ensinando a ensinar: as quatro etapas de uma aprendizagem. Editora UTFPR.

Vaillant, D., \& Marcelo, C. (2016). El ABC y D de la formación docente. Narcea.

Veiga, I. (2008). Organização didática da aula: um projeto colaborativo de ação imediata. In I. P. A. Veiga (Org.), Aula: gênese, dimensóes, princípios e práticas (pp. 40-56). Papirus.

Zeichner,Z. (2008). Uma análise crítica sobrea "reflexão" como conceito estruturante na formação docente. Educação \& Sociedade, 29(103), 535-554. 


\section{Notas}

${ }^{1}$ Contribuyeron con la difusión del cuestionario los profesores Lélia Cristina Silveira de Moraes (UFMA); Giseli Cruz (UFRJ); Luciane Maria Schlindwein (UFSC); Rita Buzzi Rauch (FURB); Marielda Ferreira Pryjma (UTFPR); Claudio Pinto Nunes (UESBE), Maria Iolanda Fontana (UTP); Marly Macedo (UFPI); Dalva Araújo; Martha Nörnberg (UFPEL); Ilma Passos Alencastro Veiga (UNB); Rosana Fernandes (UNB); Katia Curado (UNB); Chisthiany Barreiro (IFRGS); Susana Tozetto (UEPG), los miembros de los Grupos de Investigación Práxis Educativa: Dimensöes e Processos (PUCPR), Educação, Ciência e Tecnologia (UNINTER), y el Grupo de Estudos e Pesquisas - Didática e Formação Docente (UEPG). 